\title{
SYNCHRONY BREAKING BIFURCATIONS IN SMALL NEURONAL NETWORKS
}

\author{
DIKO J. HEMMINGA* \\ SPONSOR: BOB W. RINK ${ }^{\dagger}$ \\ DEPARTMENT OF MATHEMATICS \\ VRIJE UNIVERSITEIT AMSTERDAM
}

\begin{abstract}
We investigate synchrony breaking bifurcations in neuronal networks. These bifurcations occur from synchronous steady-states. In the mutual dyad and a three-neuron feed-forward chain we show that the generic bifurcation behaviour can be derived from the physical modelling parameters, in particular from the sign of the interaction between neurons. Each neuron is equipped with a simplified FitzHugh-Nagumo model and the coupling is based on synaptic coupling. An inhibitory or excitatory coupling can determine if the bifurcation is 'soft' (supercritical) or 'hard' (subcritical). For the analysis of the three-neuron feed-forward chain we follow the work of Rink and Sanders (2013): we can relate excitatory and inhibitory coupling to a 'soft' and a 'hard' transition, respectively. For the mutual dyad system we make use of a centre manifold reduction to find the type of pitchfork bifurcation. As we find an expression in terms of physical parameters, we can state whether the bifurcation is subcritical or supercritical in the weak coupling limit, and for slow and fast input.
\end{abstract}

1. Introduction. We will consider neuronal networks as dynamical systems with a network structure. The nodes of the network model the neurons. Real life neuronal networks are immensely complex but in this paper we will consider small networks. Such small networks are called 'motifs' and are often present as sub-networks of large neuronal networks. In this paper we consider the mutual dyad and a feedforward chain. The mutual dyad contains two identical neurons, mutually coupled by identical coupling. The feed-forward chain contains three identical neurons with a specific feed-forward coupling. These two motifs are shown in figures 1.1 and 1.2.

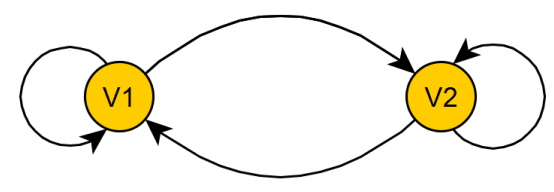

FIGURE 1.1. Mutual dyad network: two identical neurons with identical coupling.

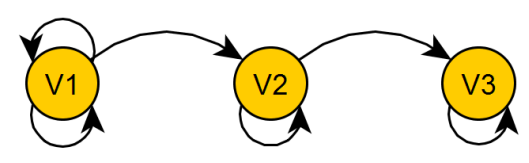

Figure 1.2. Feed-forward network of three identical neurons.

The state of a neuron can be modelled by several state variables, where the most important variable is the membrane potential. Neurons are often connected by synapses, providing communication via neurotransmitters. Such a set of connected neurons can be described by a coupled dynamical system. We model the time-dependence of the membrane potentials explicitly. We add the interaction between neurons by means of a current in the equation for the membrane potential. This results in a dynamical system given by an ordinary differential equation (ODE): a FitzHugh-Nagumo type equation.

In the theory of dynamical systems with a network structure, the generic behaviour of the motifs is known. In this paper we will take a specific set of equations to model

\footnotetext{
*Electronic address: d.j.hemminga@student.vu.nl

†Electronic address: b.w.rink@vu.nl
} 
the membrane potential of each of the neurons in a motif. We will find restrictions on the generic behaviour of these networks based on the modelling equations. This will reveal which generic behaviour really occurs in our explicit model. In this paper the sign of the interaction between neurons distinguishes the types of generic behaviour.

More specifically, we will examine bifurcations from synchronous steady-states. Such bifurcations are also called synchrony breaking bifurcations. In a coupled dynamical system we speak of a synchronous steady-state if the system is in equilibrium and the values of the state variables of the cells are equal. A bifurcation occurs if the stability of a steady-state changes by changing a parameter value. This paper contributes to answering the question: Does there exist a relation between the sign of interactions between the nodes of a network and its generic dynamics and bifurcations? Answering this question is also interesting outside of neuroscience, for general networks. In an ideal case we would be able to predict the dynamics and bifurcations in a network from the nature of the interactions between nodes.

The sign of an interaction between two neurons can be defined in terms of the dependence of the evolution of the membrane potential $V$ of one neuron on the membrane potential of the other neuron. So the sign of the interaction between cell $i$ and cell $j$ can be seen as the sign of a partial derivative

$$
\left.\frac{\partial \dot{V}_{i}}{\partial V_{j}}\right|_{V_{i}=V_{0}}
$$

In our analysis we will work with a simplified FitzHugh-Nagumo model for the individual neurons. The coupling between the neurons will be described by a simplified model for synaptic coupling. We assume that a neuron is dependent on at most one other neuron. We simplify the system of the FitzHugh-Nagumo equations including synaptic coupling to a scalar model (one-dimensional) such that bifurcation analysis is relatively easy. This work is done in section 2 .

The result is an equation for the membrane potential of a single neuron depending on its coupling to one other neuron. We use subscripts to distinguish between the two neurons conform the terminology of synapses. The differential equation describes the evolution of the potential of the 'postsynaptic' neuron situated 'after the synapse', denoted $V_{\text {post }}$. This neuron receives input via the synapse from the 'presynaptic' neuron situated 'before the synapse'. Its membrane potential is denoted by $V_{\text {pre }}$. The equation for the time dependence of the postsynaptic neuron is

$$
\dot{V}_{\text {post }}=V_{\text {post }}\left(V_{\text {post }}-a\right)\left(1-V_{\text {post }}\right)-g \cdot k\left[V_{\text {pre }}-V_{T}\right] \cdot\left(V_{\text {post }}-\nu\right)
$$

The right-hand side of eq. (1.2) consist of two parts. The first part, the internal term, is a bistable term depending on $V_{\text {post }}$, where $0<a<1$. Without the second part this term results in three equilibria $V_{\text {post }}=0, a, 1$, which are a stable, a unstable and a stable equilibrium, respectively. The second part, the coupling term, is the term corresponding to the input current, which is both dependent on $V_{\text {post }}$ and $V_{\text {pre }}$. The parameter $g$ models the conductivity, so weak coupling can be described by the limit of $g \rightarrow 0$. 
The input of the presynaptic neuron to the postsynaptic neuron is modelled by a sigmoidal function $k$. Its argument is dependent on $V_{\text {pre }}$ and $V_{T}$. The parameter $V_{T}$ corresponds to a certain threshold potential. We take $k$ proportional to a certain increasing sigmoidal function with one parameter $(p)$, which is given by

$$
k[x] \propto \frac{1}{1+\exp (-p x)} .
$$

We choose this increasing sigmoidal function to describe saturation by the input current: the synaptic current is bounded by a maximal value. The expression of the input current is also dependent on a parameter $\nu$, called the reversal potential. The difference between excitatory and inhibitory input is determined by its value. Consider the resting potential $V_{\text {rest }}$ for which the neuron is 'at rest' or in steady-state mathematically. If the reversal potential $\nu$ makes $V_{\text {rest }}-\nu$ positive, then the coupling is inhibitory (at $V_{\text {rest }}$ ). If $V_{\text {rest }}-\nu$ is negative, then the coupling is excitatory. We can actually make the sign of the interaction explicit. In terms of $V_{\text {post }}$ and $V_{\text {pre }}$ we have

$$
\left.\frac{\partial \dot{V}_{\text {post }}}{\partial V_{\text {pre }}}\right|_{V_{\text {post }}=V_{\text {pre }}=V_{\text {rest }}}=-g \cdot k^{\prime}\left[V_{\text {rest }}-V_{T}\right] \cdot\left(V_{\text {rest }}-\nu\right) .
$$

We stress that the sign of the interaction is a local quantity: we take the derivative in a point. This quantity is particularly important if we evaluate it in a bifurcation point. The sign may be both positive and negative, as it is determined by $\left(V_{\text {rest }}-\nu\right)$.

In section 3 we consider a feed-forward chain of three identical neurons with identical coupling. Each neuron is equipped with an equation for its membrane potential as given in eq. (1.2). We follow the analysis of Rink and Sanders [16]. The system can show a synchrony-breaking bifurcation of a 'soft' or 'hard' type. For the soft type there exists a stable steady state close to the synchronous steady-state after the bifurcation, but for the hard type there does not. In other words 'soft' and 'hard' transitions are distinguished by the order of the jump in the response of the system, which is of order $\mathcal{O}(1)$ and $\mathcal{O}(1)$, respectively. This can be seen in figures 1.3 (soft) and 1.4 (hard). The difference is dependent on one certain Taylor coefficient, which is calculated explicitly. We find that this Taylor coefficient is directly dependent on the sign of the coupling between neurons. Our result is summarised in the following theorem.

THEOREM 1.1. Consider the feed-forward chain with three identical neurons, described by simplified FitzHugh-Nagumo equations (eq. (1.2)). Assume that a synchronous steady state undergoes a generic synchrony breaking bifurcation. If the coupling between neurons is excitatory, the system shows a 'soft' transition (figure 1.3) and when the coupling is inhibitory, a 'hard' transition is found (figure 1.4).

In section 4 we consider the mutual dyad. We apply a bifurcation analysis to the two-dimensional system. In this network the situation is more delicate than for the feed-forward network. In view of the $\mathbb{Z}_{2}$ symmetry of the mutual dyad, its generic synchrony-breaking bifurcation is a pitchfork bifurcation. Our analysis of this bifurcation is based on centre manifold reduction. This method provides a reduced expression for the dynamics around the synchronous steady-state. We can determine 


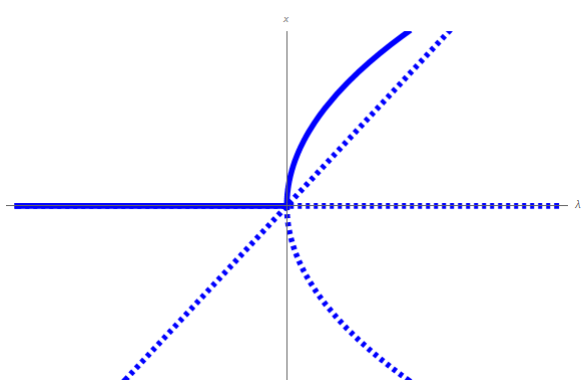

FIGURE 1.3. Bifurcation in the feedforward chain of the soft type

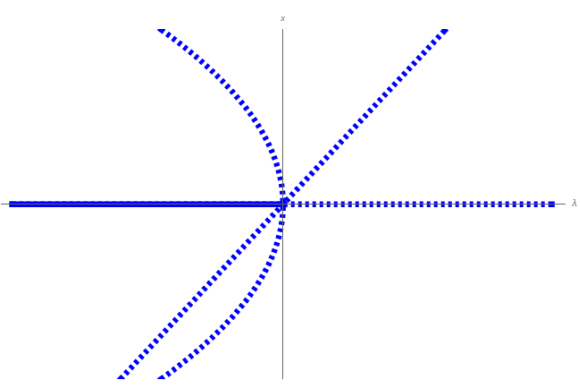

Figure 1.4. Bifurcation in the feedforward chain of the hard type

if the pitchfork bifurcation is subcritical or supercritical. These different pitchfork bifurcations can be seen in figures 1.5 and 1.6. The subcritical and supercritical pitchfork bifurcations also correspond to a 'hard' and a 'soft' transition, respectively.

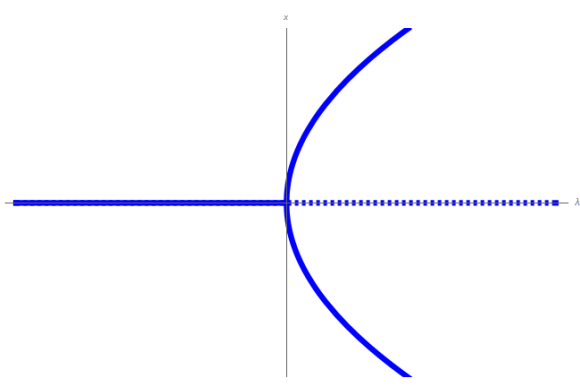

FIGURE 1.5. Supercritical pitchfork bifurcation, $x^{\prime}=c_{1} x \lambda+c_{2} x^{3}$ for positive $c_{1}$ and negative $c_{2}$.

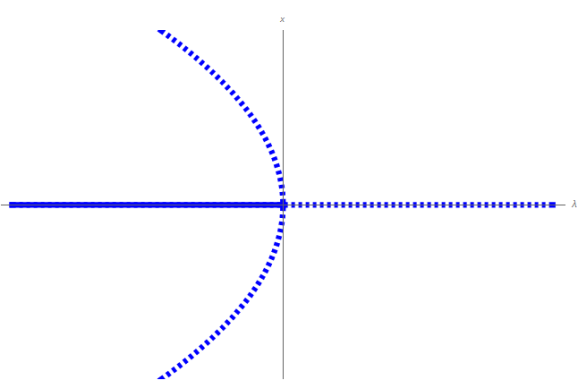

Figure 1.6. Subcritical pitchfork bifurcation, $x^{\prime}=c_{1} x \lambda+c_{2} x^{3}$ for positive $c_{1}$ and positive $c_{2}$.

To determine whether the transition is 'hard' or 'soft' we calculate the determinant Taylor coefficient in three different limits. First, we look at the case of weak coupling between the neurons. The steady states then lie close to the steady states of the uncoupled system, which are $V=0, a, 1$. The result is formulated in the following lemma.

LEMMA 1.2. In the weak coupling limit, corresponding to $g \rightarrow 0$, a generic synchrony breaking bifurcation originating from the synchronous steady state $V^{*} \approx 0$ will be a subcritical pitchfork bifurcation. For $V^{*} \approx a$ we find a supercritical pitchfork bifurcation and for $V^{*} \approx 1$ again a subcritical pitchfork bifurcation. So in this limit we find no relation between the sign of the coupling and the type of pitchfork bifurcation.

The other two limits apply to the sigmoidal function $k$, which is used to define the coupling between two neurons. In the limit $p \rightarrow 0$, corresponding to a slowly changing input, we find a delicate set of implicit conditions determining the type of pitchfork bifurcation. This is summarised in the following lemma. 
LEMMA 1.3. In the slow coupling limit, corresponding to $p \rightarrow 0$, a generic synchrony breaking bifurcation is classified based on an implicit set of conditions. The steady states $V^{*}$ in this limit solve $V^{*}\left(V^{*}-a\right)\left(1-V^{*}\right)-g \cdot\left(V^{*}-\nu\right) \cdot \frac{1}{2} \frac{\alpha}{\alpha+\beta}=0$. If we write

$$
N\left(V^{*} ; a, \nu\right)=12\left(V^{*}\right)^{3}-9\left(V^{*}\right)^{2}(1+a+\nu)+2 V^{*}(1+a)(1+a+3 \nu)-\nu\left(2+a+2 a^{2}\right)
$$

and

$$
Q\left(V^{*} ; a, \nu\right)=-2\left(V^{*}\right)^{3}+\left(V^{*}\right)^{2}(1+a+3 \nu)-2 V^{*}(1+a) \nu+a \nu,
$$

we can summarise the conditions as follows.

The synchrony breaking bifurcation is subcritical if $N\left(V^{*} ; a, \nu\right)$ and $Q\left(V^{*} ; a, \nu\right)$ have opposite sign and supercritical if $N\left(V^{*} ; a, \nu\right)$ and $Q\left(V^{*} ; a, \nu\right)$ have the same sign.

In the limit $p \rightarrow \infty$, we find that the type of pitchfork bifurcation is again directly dependent on the sign of the coupling. The results are summarised in the following theorem.

THEOREM 1.4. Consider the mutual dyad network of two identical neurons, described by simplified FitzHugh-Nagumo equations where $g \neq 0$ and $p \rightarrow \infty$. Assume that the synchronous steady state undergoes a generic synchrony breaking bifurcation. If the coupling between the neurons is excitatory, the type of pitchfork bifurcation is subcritical (a hard transition), and when the coupling is inhibitory, the type is supercritical (a soft transition).

We note that Theorem 1.1 and Theorem 1.4 state quite opposite results. Whereas Theorem 1.1 (for the feed-forward chain) connects a soft transition with excitatory coupling and a hard transition with inhibitory coupling, this connection is precisely opposite in Theorem 1.4 for the mutual dyad network.

This paper shows that by using a simplified form of the FitzHugh-Nagumo equations for the membrane potential, it is possible to investigate synchrony breaking bifurcations in small neuron networks analytically. We see that from the parameters describing the neuron and its coupling to other neurons, we can find the type of bifurcation that occurs. In a mutual dyad we can determine if the bifurcation is a subcritical or supercritical pitchfork bifurcation for each synchronous steady state. In a feed-forward chain we can also distinguish between a 'soft' and a 'hard' transition. In the case of the feed-forward chain and in the limit of $p \rightarrow \infty$ in the mutual dyad system, the difference between excitatory or inhibitory coupling determines the type of bifurcation. However, the relation is exactly opposite.

\section{Neuron modelling.}

2.1. Neuroscience. A neuron is a special cell type, with the function to transport electric signals through the body. The fundamental quantity is the membrane potential. The membrane potential is defined as the electric voltage, or potential difference, between the inside and outside of the cell membrane [4]. Connected neurons influence each others potential. This forms the basis of action potentials or 'spikes', carrying neural signals. If a neuron is not spiking, it is said to be 'in rest'. 
The potential difference is caused by the different ion concentrations in the cell. This potential is maintained by active transport of ions across the cell membrane and the selective permeability of the membrane to these ions. The most important ions are $\mathrm{Na}^{+}, \mathrm{K}^{+}$and $\mathrm{Cl}^{-}$. The ions can travel through the membrane by channels. The cell membrane itself is impermeable to ions. The channels can be gated or non-gated. If the probability of opening depends on the membrane potential, the gated channels are called voltage-gated channels. Most of these channels are closed at rest. An action potential is generated when these channels open and allow ions to flow across the cell membrane, causing a spike in the membrane potential.

Neurons can influence each other by synapses. These limbs contain gates for neurotransmitter. These small chemicals work on the ion gates of the neurons next to the synapse. Depending on the type of ion this can lead to either an increase or decrease in membrane potential. If the membrane potential is increased by the neurotransmitter, we call the neurotransmitter excitatory. If the membrane potential is decreased, the neurotransmitter is inhibitory. We use the same terminology for coupling between two neurons.

2.2. The Hodgkin-Huxley model. Hodgkin and Huxley published a paper describing the giant squid axon in 1952 [10]. This founded the field of mathematical neuroscience. They proposed a four-dimensional system of differential equations for the dynamics of the membrane potential and three gating variables. The HodgkinHuxley model is based on the dependence of the membrane voltage on the ion currents crossing the neuron cell membrane. The ions can travel through the cell membrane by voltage-gated channels. Thus the differential equation for the membrane potential is dependent on gating variables. The precise coefficients have been derived by Hodgkin and Huxley from experimental data of measurements of the giant squid axon. Their research was highly recognized by the scientific community: they were awarded the Nobel prize in Physiology or Medicine in 1963 [14].

2.3. The FitzHugh-Nagumo model. FitzHugh stated a neuron model in 1961, based on the Hodgkin-Huxley equations with some large simplifications. It is a two-dimensional model developed to capture the essence of the Hodgkin-Huxley equations. The model is based on a modification of the Van der Pol equation. So FitzHugh called it the "Bonhoeffer-Van der Pol model" [5, 6]. The equivalent is an electrical circuit developed by Nagumo in 1962 [13]. The system of equations for the FitzHugh-Nagumo model is given in eq. (2.1). The membrane potential $V$ shows bistable behaviour. The variable $w$ is called a recovery variable.

By an input current $I$, the membrane potential can be influenced by an external applied current or the membrane potentials of other neurons. This will be discussed in section 2.4. The recovery variable is modelled as slow with respect to the membrane potential $V$, so the positive parameter $\epsilon$ is assumed to be small [4]. The goal of the FitzHugh-Nagumo model is to isolate conceptually the essential mathematical properties of excitability. Excitability means that the model can describe a 'firing' neuron: a peak in the membrane voltage. The Hodgkin-Huxley model describes this by the electrochemical properties of sodium and potassium ion flow. While this makes the Hodgkin-Huxley model more realistic, the basic properties of excitability are explained by the FitzHugh-Nagumo model. Although not clearly derivable from biology, the FitzHugh-Nagumo model allows for simplified dynamics, without being a trivial simplification [11]. 
In this paper we base our model for one neuron on the FitzHugh-Nagumo equations. The system of equations for the FitzHugh-Nagumo model is

$$
\begin{aligned}
& \frac{\mathrm{d} V}{\mathrm{~d} t}=V(V-a)(1-V)-w+I, \\
& \frac{\mathrm{d} w}{\mathrm{~d} t}=\epsilon(V-\gamma w),
\end{aligned}
$$

where $0<a<1, \epsilon>0$, and $\gamma \geq 0$ [4]. Our main reason for the choice of this model is that it is low-dimensional to keep the analysis manageable. The FitzHugh-Nagumo model is two-dimensional, compared to the four-dimensional Hodgkin-Huxley model. However, by isolating the essential mathematical components it displays several features of neurons. The Hodgkin-Huxley model is biologically more precise, but needs biological data and computer simulations to be analysed. In this paper we consider small networks of neurons instead of a single neuron. This implies that keeping the equations for one neuron manageable is more important than the biological correctness for one neuron.

2.4. Modelling input current by synaptic coupling. The parameter $I$ in the first equation of the system (2.1) is called an input current. The coupling between neurons is implemented assuming synaptic connections. Chemical synapses are the communication devices between neurons. They excrete neurotransmitter to influence receptors on other neurons. The mathematical implementation is as follows [4]: We define the synaptic input current $I_{\text {syn }}$ as

$$
I_{\text {syn }}:=g_{\text {syn }} \cdot s(t) \cdot\left(V_{\text {post }}-\nu_{\text {syn }}\right),
$$

where $s(t)$ is the fraction open channels. Let us call the voltage variable $V_{\text {post }}$, the membrane potential of the postsynaptic cell. The postsynaptic cell receives the input current. The parameter $g_{s y n}$ is a factor with an interpretation of conductivity. The parameter $\nu_{\text {syn }}$ is called the synaptic reversal potential. It is the trans-membrane voltage at which the diffusive and electrical forces counterbalance. Whether a synapse is excitatory or inhibitory is dependent on this parameter, see the introduction. The terms excitatory and inhibitory are defined in biology in this manner: An excitatory input increases the membrane potential, an inhibitory decreases the membrane potential.

We assume that the fraction of open channels, $s=s(t)$, satisfies the differential equation

$$
\frac{\mathrm{d} s}{\mathrm{~d} t}=\alpha(1-s) \cdot H\left(V_{p r e}-V_{T}\right)-\beta s,
$$

Eq. (2.3) is an example of a gate model. This equation is dependent on the presynaptic membrane potential, $V_{p r e}$, and some threshold potential, $V_{T}$. In this equation $\alpha$ and $\beta$ are the rates at which the synapse turns on and off, respectively, modelled by the rate of opening and closing of the neurotransmitter gates. In eq. (2.3) $H$ represents a smooth approximation of a Heaviside function. The true Heaviside function satisfies $H_{\infty}\left(V_{\text {pre }}-V_{T}\right)=0$ if $V_{\text {pre }}-V_{T}<0$ and $H_{\infty}\left(V_{\text {pre }}-V_{T}\right)=1$ if $V_{\text {pre }}-V_{T}>0$ and a smooth and increasing approximation is often called a sigmoidal function, which has the same limiting behaviour, but is smooth around $V_{\text {pre }}-V_{T}=0$. 
Now that the equations for the coupling between neurons are established, we state the complete set of modelling equations for one neuron. To get the correct excitatory/inhibitory behaviour we set $I=-I_{\text {syn }}$ in the FitzHugh-Nagumo equations (eq. (2.1)). Each neuron is described by a set of equations such as given in (2.4). These equations describe a neuron with membrane potential $V_{\text {post }}$ and state variables $w$ and $s$. The equations are

$$
\begin{cases}\frac{\mathrm{d} V_{\text {post }}}{\mathrm{d} t} & =V_{\text {post }}\left(V_{\text {post }}-a\right)\left(1-V_{\text {post }}\right)-w-g_{\text {syn }} \cdot s(t) \cdot\left(V_{\text {post }}-\nu_{\text {syn }}\right), \\ \frac{\mathrm{d} w}{\mathrm{~d} t} & =\epsilon \cdot\left(V_{\text {post }}-\gamma w\right), \\ \frac{\mathrm{d} s}{\mathrm{~d} t} & =\alpha(1-s) \cdot H\left(V_{\text {pre }}-V_{T}\right)-\beta s .\end{cases}
$$

2.5. Reductions. The parameter $\epsilon$ is small, which makes the changes in $w$ slow with respect to $V$. Unfortunately, this means that the assumption that $w$ is always in equilibrium does not hold. If $w$ would change fast with respect to $V$, the dynamics of $V$ would find $w$ always close to equilibrium. The simplification we make for $w$ is that we approximate $w=0$ and $w^{\prime}=0$ which simplifies both the first and second equation in eq. (2.4). This dimensional reduction is necessary to be able to carry out the analysis in chapters 3 and 4.

For the second simplification we eliminate the differential equation for $s$ and write $s=\bar{s}\left(V_{\text {pre }}-V_{T}\right)$ in the equation for $V_{\text {post }}$. Here $\bar{s}$ denotes the steady-state of eq. $(2.3)$. It is easy to find this steady-state for eq. (2.3), that is the value of $s$ such that $\frac{\mathrm{d} s}{\mathrm{~d} t}=0$. This result in

$$
\bar{s}=\bar{s}\left(V_{\text {pre }}-V_{T}\right)=\frac{\alpha H}{\alpha H+\beta}= \begin{cases}0 & \text { if } V_{\text {pre }}-V_{T}<0 \\ \frac{\alpha}{\alpha+\beta} & \text { if } V_{\text {pre }}-V_{T}>0\end{cases}
$$

where the last equality only follows if $H_{\infty}$ is the true Heaviside function.

The function $\bar{s}\left(V_{\text {pre }}-V_{T}\right)$ can be approximated by a sigmoidal function dependent on $V_{\text {pre }}$. A simple form of a sigmoidal function will be used here, which is known as the logistic function $k_{p}(x)$

$$
k(x)=k_{p}(x)=\frac{\alpha}{\alpha+\beta} \frac{1}{1+\exp (-p x)}
$$

with parameter $p$. The logistic function is one from a variety of sigmoidal functions that have been used as activation function of artificial neurons [12]. Concluding, we define $s=\bar{s}\left(V_{\text {pre }}-V_{T}\right)=k_{p}\left(V_{\text {pre }}-V_{T}\right)$.

This simplification, by using the steady-state approximation of $s$, makes bifurcation analysis possible, as the explicit equations are only one-dimensional. This means that the equation for membrane potential $V_{\text {post }}$ is directly dependent on membrane potential $V_{\text {pre }}$. So the terminology 'direct coupling' is introduced after this simplification. After these two simplifications the system of equations in eq. (2.4) is reduced to a single equation as the model for one neuron,

$$
\dot{V}_{\text {post }}=V_{\text {post }}\left(V_{\text {post }}-a\right)\left(1-V_{\text {post }}\right)-g \cdot k\left[V_{\text {pre }}-V_{T}\right] \cdot\left(V_{\text {post }}-\nu\right) .
$$




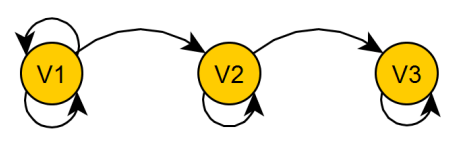

FIGURE 3.1. Schematic representation of a homogeneous feed-forward network consisting of three neurons.

3. Three-neuron feed-forward chain. In this section we consider the threecell feed-forward motif. The name is derived from the fact that this network is a chain of coupled neurons, without feedback to neurons earlier in the chain. Feed-forward chains occur often as 'motifs' in larger networks, because a feed-forward chain can act as an amplifier [9]. This behaviour is proved mathematically in Rink and Sanders (2013) as they found faster amplitude growth for state variables further in the chain $[15,16]$. Figure 3.1 shows the network under consideration, a homogeneous feedforward chain of three neurons.

We consider a chain of three neurons as shown in figure 3.1, where the response function for the membrane potential is of the form of eq. (2.7). The system of equations for the three neurons in the feed-forward network will be

$$
\begin{aligned}
& \dot{V}_{1}=f\left(V_{1}, V_{1}\right)=V_{1}\left(V_{1}-a\right)\left(1-V_{1}\right)-g \cdot k\left[V_{1}-V_{T}\right] \cdot\left(V_{1}-\nu\right), \\
& \dot{V}_{2}=f\left(V_{2}, V_{1}\right)=V_{2}\left(V_{2}-a\right)\left(1-V_{2}\right)-g \cdot k\left[V_{1}-V_{T}\right] \cdot\left(V_{2}-\nu\right), \\
& \dot{V}_{3}=f\left(V_{3}, V_{2}\right)=V_{3}\left(V_{3}-a\right)\left(1-V_{3}\right)-g \cdot k\left[V_{2}-V_{T}\right] \cdot\left(V_{3}-\nu\right) .
\end{aligned}
$$

In these equations the simplified FitzHugh-Nagumo model with direct coupling is used, so $k$ denotes the sigmoidal function defined in eq. (2.6). This system of equations is a special case of a general feed-forward network [16]. The system is studied in Rink and Sanders [16] and is often encountered in the literature on coupled cell networks $[3,7,8,9]$.

3.1. Analysis of the feed-forward system. We begin the analysis by stating a modified form of Theorem 2.3 in Rink and Sanders [16]. The theorem stated in Rink and Sanders [16] is valid for a more general class of $n$-neuron feed-forward networks with one parameter $\lambda$. In our situation any of the parameters $a, g, p, \nu$ can play the role of $\lambda$.

TheOREM 3.1 (Modified from Theorem 2.3 in Rink and Sanders [16]). Let $\hat{f}: \mathbb{R}^{2} \times \mathbb{R} \rightarrow \mathbb{R}$ be a smooth function. Assume that $\hat{f}(0 ; \lambda)=0$. Here we denote $a_{i}=a_{i}(\lambda):=D_{i} \hat{f}(0 ; \lambda)$ for $a=1,2[16]$. Assume that $a_{1}(0)=0$. Furthermore, assume the following generic conditions on $\hat{f}(X ; \lambda)$ :

1. $a_{1}(0)+a_{2}(0) \neq 0$,

2. $\frac{\mathrm{d} a_{1}}{\mathrm{~d} \lambda}(0) \neq 0$,

3. $a_{2}(0) \neq 0$,

4. $\frac{\partial^{2} f}{\partial X_{1}^{2}}(0 ; 0) \neq 0$. 
Then the equations

$$
\begin{aligned}
& \dot{x}_{1}=\hat{f}\left(x_{1}, x_{1} ; \lambda\right), \\
& \dot{x}_{2}=\hat{f}\left(x_{2}, x_{1} ; \lambda\right), \\
& \dot{x}_{3}=\hat{f}\left(x_{3}, x_{2} ; \lambda\right),
\end{aligned}
$$

support 4 branches of hyperbolic steady states (counted excluding the trivial steady state $x=0)$ near $(x ; \lambda)=(0 ; 0)$. More precisely, there are two steady state branches with asymptotics

$$
x_{1}=x_{2}=0, x_{3} \sim|\lambda|,
$$

and two with asymptotics

$$
x_{1}=0, x_{2} \sim|\lambda|, x_{3} \sim|\lambda|^{\frac{1}{2}}
$$

From Remark 2.4 in Rink and Sanders [16] we conclude that only one of the branches proportional to $\sqrt{\lambda}$ can be stable while the branches proportional to $\lambda$ are never stable. Remark 2.4 in Rink and Sanders [16] states that this happens if $a_{1}(0)+a_{2}(0)<0$ and $a_{2}(0)>0$. We call the transition a 'soft' transition if a stable branch exists on both sides of $\lambda=0$. So we see that a 'soft' transition, as shown in figure 3.2, corresponds to a positive sign of $a_{2}(0)$. When $a_{2}(0)<0$ the bifurcation diagram shows a 'hard' transition, which is shown in figure 3.3.

In the paper by Rink and Sanders (2013) the (general) response function $\hat{f}$ satisfies $\hat{f}(0,0,0, \lambda)=0$ for all $\lambda$, which implies that the equations describing the system admit a fully synchronous steady state solution $(0,0,0)$ for all values of $\lambda$. This does not hold for $f$ in eq. (3.1). However, as the condition $\partial_{1} f(0,0,0)+\partial_{2} f(0,0,0) \neq 0$ holds, we find that by the Inverse Function Theorem there exists a synchronous steady state $V^{*}$.

This synchronous steady state $V^{*}$, that is $V_{1}=V_{2}=V_{3}=V^{*}(\lambda)$, satisfies $f\left(V^{*}, V^{*}, \lambda\right)=$ 0 . To accommodate the condition in Theorem 3.1, $\hat{f}(0,0,0, \lambda)=0$, we define new variables $x_{1}, x_{2}, x_{3}$ as $x_{1}:=V_{1}-V^{*}(\lambda), x_{2}:=V_{2}-V^{*}(\lambda)$ and $x_{3}:=V_{3}-V^{*}(\lambda)$. This results in the redefinition of the response function $f$ from eq. (3.1),

$$
\begin{aligned}
& \hat{f}\left(x_{1}, x_{2}, \lambda\right)= \\
& \left(x_{1}+V^{*}\right)\left(\left(x_{1}+V^{*}\right)-a\right)\left(1-\left(x_{1}+V^{*}\right)\right)-g \cdot k\left[\left(x_{2}+V^{*}\right)-V_{T}\right] \cdot\left(\left(x_{1}+V^{*}\right)-\nu\right) .
\end{aligned}
$$

so that the condition $\hat{f}(0,0,0, \lambda)=0$ for all $\lambda$ holds. Hence $x=\left(x_{1}, x_{2}, x_{3}\right)=(0,0,0)$ is a fully synchronous steady state solution for the system.

3.2. Bifurcations for the three-neuron feed-forward chain. Assume there is a synchronous steady state $V_{1}=V_{2}=V_{3}=V^{*}$ for the homogeneous feed-forward chain consisting of three neurons. The bifurcation behaviour of the system of equations based on eq. (3.7) can be described as a soft (figure 3.2) or a hard (figure 3.3) transition for small changes of $\lambda$ around 0 . As will be shown below, the type of transition is directly dependent on the sign of the coupling between the neurons. This result is summarized in the following theorem. 
THEOREM 3.2. Consider the system of the feed-forward chain with three identical neurons, described by simplified FitzHugh-Nagumo equations. Assume that a synchronous steady state undergoes a generic synchrony-breaking bifurcation as one of the parameters $a, g, p, \nu$ is varied. If the coupling between cells is excitatory at the bifurcation, the system shows a 'soft' transition and when the coupling is inhibitory, a 'hard' transition is found.

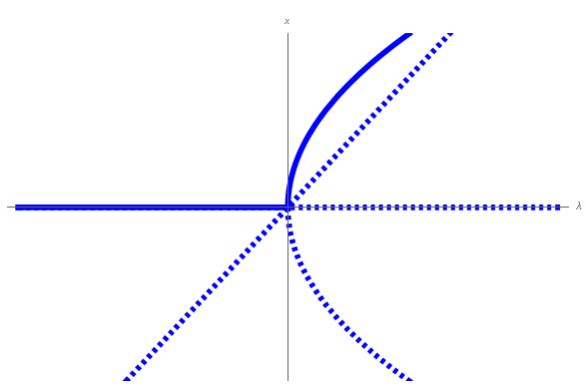

Figure 3.2. Bifurcation in the feedforward network of the soft type

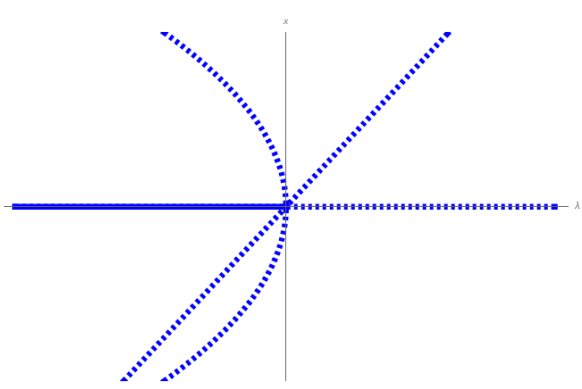

Figure 3.3. Bifurcation in the feedforward network of the hard type

Proof. From Remark 2.4 in Rink and Sanders [16] we find that $a_{2}(0)$ determines if the transition is 'soft' $\left(a_{2}(0)>0\right.$, figure 3.2) or 'hard' $\left(a_{2}(0)<0\right.$, figure 3.3) The expression for the response function is explicitly known, as stated in eq. (3.7). So we

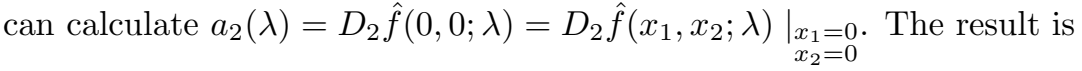

$$
a_{2}(\lambda)=\frac{\partial \hat{f}\left(x_{1}, x_{2}, \lambda\right)}{\partial x_{2}}=-g \cdot k^{\prime}\left[x_{2}+V^{*}-V_{T}\right] \cdot\left(x_{1}+V^{*}-\nu\right),
$$

which gives

$$
\left.\frac{\partial \hat{f}\left(x_{1}, x_{2}, \lambda\right)}{\partial x_{2}}\right|_{\substack{x_{1}=0 \\ x_{2}=0}}=-g \cdot k^{\prime}\left[V^{*}-V_{T}\right] \cdot\left(V^{*}-\nu\right),
$$

The signs of $g$ and $k^{\prime}$ are both positive, based on their physical meaning, see section 2 . This makes the sign of the expression completely dependent on the sign of the expression $V^{*}-\nu$. The interpretation of this sign is clear from the physical meaning of the parameter $\nu$, as mentioned in the Introduction. For a inhibitory coupling between two neurons, the sign of $V^{*}-\nu$ is positive, which implies that $a_{2}(\lambda)<0$, corresponding to a hard transition in the bifurcation diagram (figure 3.3). If the coupling is excitatory, the sign of $V^{*}-\nu$ is negative, which implies that $a_{2}(\lambda)>0$, corresponding to a soft transition in the bifurcation diagram (figure 3.2).

4. Mutual dyad. In this final chapter we consider the system of the mutual dyad with the simplified system of equations for two identical neurons. The differential equations for the membrane potentials are directly dependent on each other, by means of the sigmoidal function $k$. We find the following system of equations

$$
\begin{aligned}
& \dot{V}_{1}=V_{1}\left(V_{1}-a\right)\left(1-V_{1}\right)-g \cdot k\left[V_{2}-V_{T}\right] \cdot\left(V_{1}-\nu\right)=: f\left(V_{1}, V_{2}\right), \\
& \dot{V}_{2}=V_{2}\left(V_{2}-a\right)\left(1-V_{2}\right)-g \cdot k\left[V_{1}-V_{T}\right] \cdot\left(V_{2}-\nu\right)=: f\left(V_{2}, V_{1}\right)
\end{aligned}
$$


We can perform a bifurcation analysis on this system. The results of this section are statements about the type of pitchfork bifurcation in this system for different sets of parameters.

4.1. Bifurcation analysis. This system shows $\left(V_{1}, V_{2}\right) \leftrightarrow\left(V_{2}, V_{1}\right)$ symmetry. Assume that $\left(V_{1}, V_{2}\right)=\left(V^{*}, V^{*}\right)$ is a synchronous steady state. Now we introduce the following notation

$$
\begin{aligned}
& X=\partial_{1} f\left(V^{*}, V^{*}\right)=-3\left(V^{*}\right)^{2}+2(a+1) V^{*}-g \cdot k\left[V^{*}-V_{T}\right]-a \\
& Y=\partial_{2} f\left(V^{*}, V^{*}\right)=-g \cdot\left(V^{*}-\nu\right) \cdot k^{\prime}\left[V^{*}-V_{T}\right]
\end{aligned}
$$

for the entries of the Jacobian evaluated in the synchronous point $V_{1}=V_{2}=V^{*}$. Then the eigenvalues of the Jacobian are $X \pm Y$, where both $X$ and $Y$ can positive and negative. The result is that the corresponding eigenvectors are $(1,1)$ for the eigenvalue $X+Y$ and $(1,-1)$ for the eigenvalue $X-Y$.

We are interested in a synchrony breaking bifurcation, where the system moves away from the synchronous steady state. A bifurcation occurs if an eigenvalue goes through zero, when changing a parameter $\lambda$. The synchrony breaking bifurcation can happen if the corresponding eigenvalue is zero: $X-Y=0$ so if $X=Y$ [17].

Assume we have a synchronous steady-state where a synchrony-breaking bifurcation takes place for $V_{1}=V_{2}=V^{*}$ and $\lambda=\lambda^{*}$. We will continue the analysis with a centre manifold reduction. The centre manifold consists of orbits whose behaviour around the equilibrium point is not controlled by either attraction of the stable manifold or repulsion of the unstable manifold. In our application the centre manifold is tangent to the eigenspace of eigenvalue $X-Y$, when that eigenvalue is zero $(X=Y)$. One of the main methods of simplifying a dynamical system is to reduce the dimension of the system. Centre manifold theory is a rigorous mathematical technique that makes this reduction possible, at least near equilibria $[1,2,18]$.

We first transform the coordinates $\left(V_{1}, V_{2}\right)$ of the coupled system in eq. (4.1) to new coordinates $(x, y)$ such that the $x$-axis is the span of $(1,-1)$ and the $y$-axis is the span of $(1,1)$. We also move the point where the bifurcation takes place to the origin. Both transformations result in the definition of $x$ and $y$

$$
\begin{aligned}
& x=V_{1}-V_{2}, \\
& y=V_{1}+V_{2}-2 V^{*}(\lambda)
\end{aligned}
$$

where $V^{*}(\lambda)$ denotes the value of the synchronous equilibrium point.

So we have a synchronous steady-state at $x=0, y=0$ with the symmetry $(x, y) \leftrightarrow$ $(-x, y)$. From the equations for $V_{1}$ and $V_{2}$ we can deduce the differential equations for $x$ and $y$. So given the equations for $\dot{V}_{1}$ and $\dot{V}_{2}$ in eq. (4.1) and the transformations from $(x, y)$ to $\left(V_{1}, V_{2}\right)$ by inverting eq. (4.4) we can compose the equation for $\dot{x}$ and $\dot{y}$. 
So the equations governing the dynamics of $x$ and $y$ are

$$
\begin{aligned}
\dot{x} & =f\left(\frac{(y+x)}{2}+V^{*}, \frac{(y-x)}{2}+V^{*}, \lambda\right)-f\left(\frac{(y-x)}{2}+V^{*}, \frac{(y+x)}{2}+V^{*}, \lambda\right), \\
& =: g(x, y, \lambda), \\
\dot{y} & =f\left(\frac{(y+x)}{2}+V^{*}, \frac{(y-x)}{2}+V^{*}, \lambda\right)+f\left(\frac{(y-x)}{2}+V^{*}, \frac{(y+x)}{2}+V^{*}, \lambda\right), \\
& =: h(x, y, \lambda),
\end{aligned}
$$

where $V^{*}=V^{*}(\lambda)$.

Remember the linearisation of the system in $V_{1}$ and $V_{2}$, with $X=\partial_{1} f\left(V^{*}, V^{*}, \lambda\right)$ and $Y=\partial_{2} f\left(V^{*}, V^{*}, \lambda\right)$ as defined before, at the synchronous point $\left(V^{*}, V^{*}, \lambda\right)$. After the change of coordinates the linearised system shows the eigenvalues of the Jacobian explicitly

$$
\begin{aligned}
& \dot{x}=(X-Y) x+\text { h.o.t. }, \\
& \dot{y}=(X+Y) y+\text { h.o.t.. }
\end{aligned}
$$

A bifurcation takes place for $\lambda=\lambda^{*}$, for which $X-Y=0$ but also $X+Y \neq 0$ by assumption.

We state here the result of the centre manifold reduction in a general form. We will later derive the Taylor coefficients of the equation on the centre manifold explicitly. These expressions are dependent on a set of Taylor coefficients of the functions $g$ and $h$. In this section we will evaluate these expressions for the explicit form of $g$ and $h$ defined in terms of the response function $f$ in eq. (4.5).

Lemma 4.1. Consider the smooth system of ordinary differential equations

$$
\left\{\begin{array}{l}
\dot{x}=g(x, y, \lambda), \\
\dot{y}=h(x, y, \lambda),
\end{array}\right.
$$

where $g$ is odd in $x, \frac{\partial g}{\partial x}(0,0,0)=0$ and $\frac{\partial g}{\partial y}(0,0,0)=0$. Also $h$ is even in $x$, $h(0,0,0)=0, \frac{\partial h}{\partial x}(0,0,0)=0$ and $\frac{\partial h}{\partial y}(0,0,0) \neq 0$. By the centre manifold theorem $([1,2,18])$ there exists a smooth real-valued function $m(x, \lambda)$ such that $y=m(x, \lambda)$ is an invariant manifold and $\partial_{x} m(0,0)=0$. This manifold contains all the solutions close to 0 that remain near 0 for all $t \in \mathbb{R}$, such as equilibria and periodic solutions.

$A$ centre manifold reduction at the synchronous point $(x, y, \lambda)=(0,0,0)$ produces an equation on the centre manifold of the form

$$
\frac{\mathrm{d} x}{\mathrm{~d} t}=c_{1} x \lambda+c_{2} x^{3}+\mathcal{O}\left(|x|^{5}+|x|^{3}|\lambda|+|x||\lambda|^{2}\right),
$$

Here $c_{1}$ and $c_{2}$ can be computed from the coefficients of the Taylor expansion of $g$ and $h$ around $(x, y, \lambda)=(0,0,0)$, namely

$$
\begin{aligned}
& c_{1}=G-\frac{F \hat{D}}{\hat{C}}, \\
& c_{2}=H-\frac{F \hat{E}}{\hat{C}},
\end{aligned}
$$


where

$$
\begin{array}{r}
\hat{C}=\frac{\partial h}{\partial y}(x, y, \lambda), \hat{D}=\frac{\partial h}{\partial \lambda}(x, y, \lambda), \hat{E}=\frac{\partial^{2} h}{\partial x^{2}}(x, y, \lambda), \\
F=\frac{\partial^{2} g}{\partial x \partial y}(x, y, \lambda), G=\frac{\partial^{2} g}{\partial x \partial \lambda}(x, y, \lambda), H=\frac{\partial^{3} g}{\partial x^{3}}(x, y, \lambda) .
\end{array}
$$

The proof of this lemma omitted. It is based on calculating the Taylor expansion of the differential equation of the centre manifold. By imposing the invariance equations on expansions of $g, h$ and the equation of the centre manifold $m$, we find the stated expression of $m$ in terms of the Taylor coefficients of $g$ and $h$. We also do not prove Corollary 4.2 below which simply follows from the definitions of $f, g$ and $h$.

Corollary 4.2. From the explicit equation of $f$ in eq. (4.1) combined with the definitions of $g$ and $h$ in eq. (4.5) we can find that the explicit expression of $c_{2}$ from eq. (4.8) is equal to

$$
\begin{aligned}
H-\frac{F \hat{E}}{\hat{C}}= & \frac{1}{4}\left(-6+g \cdot\left(V^{*}-\nu\right) \cdot k^{\prime \prime \prime}\left[V^{*}-V_{T}\right]-3 g \cdot k^{\prime \prime}\left[V^{*}-v_{T}\right]\right) \\
- & \frac{1}{4} \frac{1}{-3\left(V^{*}\right)^{2}+2(a+1) V^{*}-g \cdot k\left[V^{*}-V_{T}\right]-a-g \cdot\left(V^{*}-\nu\right) \cdot k^{\prime}\left[V^{*}-V_{T}\right]} \\
& \left(\left(-6 V^{*}+2(a+1)\right)^{2}-\left(g \cdot\left(V^{*}-\nu\right) \cdot k^{\prime \prime}\left[V^{*}-V_{T}\right]\right)^{2}\right. \\
+ & \left.2 g \cdot k^{\prime}\left[V^{*}-V_{T}\right]\left(-6 V^{*}+2(a+1)+g \cdot k^{\prime}\left[V^{*}-V_{T}\right]\right)\right)
\end{aligned}
$$

4.2. Subcritical and supercritical pitchfork bifurcations. In the previous section we found that the reduced equation on the centre manifold is given by the expression in eq. (4.8). The generic bifurcation in systems with $\mathbb{Z}_{2}$ symmetry is a pitchfork bifurcation which occurs if $c_{1}, c_{2} \neq 0$. We consider a synchronous steady state $V_{1}=V_{2}=V^{*}$ which undergoes a bifurcation for $\lambda=\lambda^{*}$. Such a synchronous steady state $V^{*}$ is the solution to the equation $f\left(V^{*}, V^{*}, \lambda\right)=0$ where $V^{*}=V^{*}(\lambda)$. For our explicit $f$ this is

$$
V^{*}\left(V^{*}-a\right)\left(1-V^{*}\right)-g \cdot k\left[V^{*}-V_{T}\right] \cdot\left(V^{*}-\nu\right)=0,
$$

where we can also put in $k\left[V^{*}-V_{T}\right]$, resulting in

$$
V^{*}\left(V^{*}-a\right)\left(1-V^{*}\right)-g \cdot\left(\frac{\alpha}{\alpha+\beta}\right) \frac{1}{1+e^{-p\left(V^{*}-V_{T}\right)}} \cdot\left(V^{*}-\nu\right)=0 .
$$

A pitchfork bifurcation can occur in two different forms, the supercritical and subcritical pitchfork bifurcation. In figures 4.1 and 4.2 we show the form of the supercritical and subcritical pitchfork bifurcation, respectively. We can use the terms soft or hard transition as well. A supercritical pitchfork bifurcation corresponds to a soft transition (figure 4.1), whereas a subcritical pitchfork bifurcation corresponds to a hard transition (figure 4.2). The same terminology is used for the variants with negative $c_{1}$, corresponding to diagrams mirrored in the vertical axis.

By checking these four cases explicitly we can deduce that the sign of the coefficient $c_{2}$ 
in front of $x^{3}$ determines the type of pitchfork bifurcation. The supercritical variant occurs for a negative coefficient $c_{2}$ in front of $x^{3}$ and the subcritical variant for a positive coefficient $c_{2}$. Therefore the coefficient $H-\frac{F \hat{E}}{\hat{C}}$ is the subject for the following analysis. We already found that this coefficient can be written as in eq. (4.13).

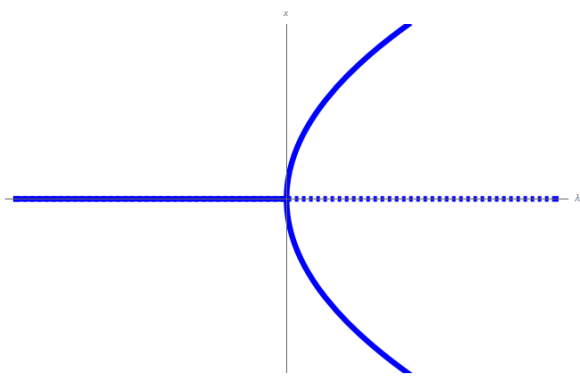

FIGURE 4.1. Supercritical pitchfork bifurcation, $x^{\prime}=c_{1} x \lambda+c_{2} x^{3}$ for positive $c_{1}$ and negative $c_{2}$.

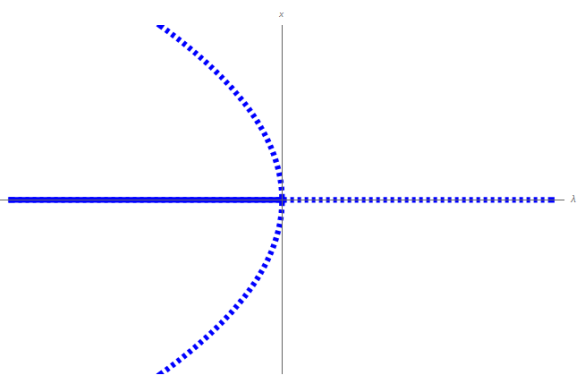

FiguRE 4.2. Subcritical pitchfork bifurcation, $x^{\prime}=c_{1} x \lambda+c_{2} x^{3}$ for positive $c_{1}$ and positive $c_{2}$.

As the expression in eq. (4.13) contains a large number of parameters, it is not possible to find all possible scenarios which result in a certain sign of the coefficient of $x^{3}$. We will simply analyse the expression in the case for certain limits of some of the parameters.

Weak coupling limit. Consider the limit where the parameter $g$ approaches zero. The physical interpretation is that the conductivity $g$ decreases, corresponding to weak coupling in biological terms. We find the expression for the relevant coefficient to determine the type of bifurcation in this limit, which states

$$
\lim _{g \rightarrow 0}\left(H-\frac{F \hat{E}}{\hat{C}}\right)=-\frac{3}{2}-\frac{1}{4} \frac{\left(-6 V^{*}+2(a+1)\right)^{2}}{\left(-3\left(V^{*}\right)^{2}+2(a+1) V^{*}-a\right)} .
$$

We consider the limit for $g$ to zero, so we assume that the steady states $V^{*}$ lie close to the steady states for $g=0$. In that case the expression for the response function $f$ reduces to a cubic polynomial, resulting in steady states $V^{*}=0, a, 1$.

For the first case of $V^{*}=0$ we find

$$
\left.\lim _{g \rightarrow 0}\left(H-\frac{F \hat{E}}{\hat{C}}\right)\right|_{V^{*}=0}=a+\frac{1}{2}+\frac{1}{a}>0,
$$

where the last inequality follows from $0<a<1$. Now for the steady state $V^{*}=a$ we find that

$$
\left.\lim _{g \rightarrow 0}\left(H-\frac{F \hat{E}}{\hat{C}}\right)\right|_{V^{*}=a}=\frac{5}{2}-\frac{1}{1-a}-\frac{1}{a}<0,
$$

again using that $0<a<1$. For $V^{*}=1$

$$
\left.\lim _{g \rightarrow 0}\left(H-\frac{F \hat{E}}{\hat{C}}\right)\right|_{V^{*}=1}=-a+\frac{3}{2}+\frac{1}{1-a}>0 .
$$


We summarise the conclusions in the following lemma.

LeMma 4.3. In the weak coupling limit, corresponding to $g \rightarrow 0$, a generic synchrony breaking bifurcation originating from the synchronous steady state $V^{*} \approx 0$ will be a subcritical pitchfork bifurcation, for $V^{*} \approx a$ we find a supercritical pitchfork bifurcation and for $V^{*} \approx 1$ again a subcritical pitchfork bifurcation. So in this limit we find no relation between the sign of the coupling and the type of pitchfork bifurcation.

Limit of slow changing input. Next, we consider taking the limit of $p \rightarrow 0$. The physical interpretation of the limit $p \rightarrow 0$ corresponds to small changes in input current for changes in potential in the inputting neuron. An example of a sigmoidal function for a small value of $p$ is shown in figure 4.3. The sigmoidal function with parameter $p$ introduced in eq. (2.6) reads

$$
k[x]=\sigma(x ; p)=\frac{\alpha}{\alpha+\beta} \frac{1}{1+\exp (-p x)} .
$$

For finding the steady states for $p \rightarrow 0$ we assume they will be close to the states we find for $p=0$. If $p=0$ we find that $k[x ; 0]=\frac{1}{2} \frac{\alpha}{\alpha+\beta}$. The steady states must solve

$$
V^{*}\left(V^{*}-a\right)\left(1-V^{*}\right)-g \cdot\left(V^{*}-\nu\right) \cdot \frac{1}{2} \frac{\alpha}{\alpha+\beta}=0 .
$$

Recall that we have the parameter bounds $0<a<1$ and $g>0$.

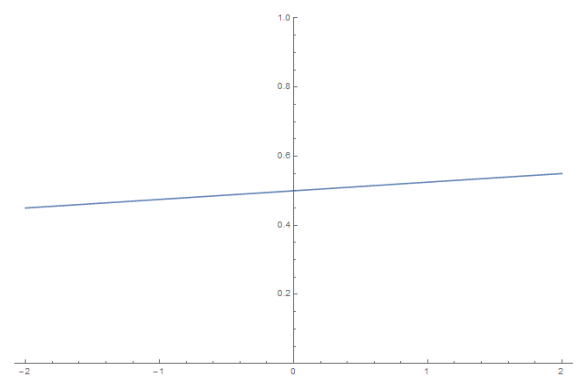

FIGURE 4.3. Sigmoidal function for $p a-$ rameter $p=\frac{1}{10}$.

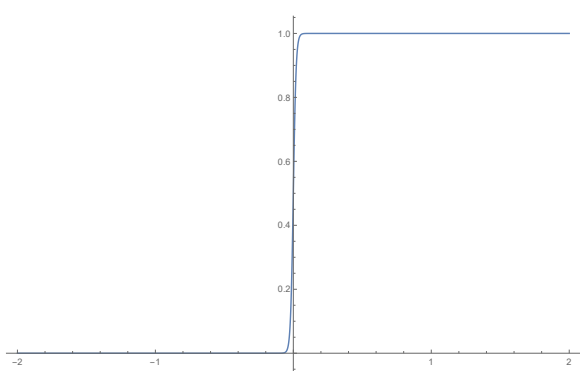

Figure 4.4. Sigmoidal function for $p a-$ rameter $p=100$

The results for the limit $p \rightarrow 0$ are stated in the following lemma.

Lemma 4.4. In the slow coupling limit, corresponding to $p \rightarrow 0$, a generic synchrony breaking bifurcation is classified based on an implicit set of conditions. The steady states $V^{*}$ in this limit solve eq. (4.19). If we write

$$
N\left(V^{*} ; a, \nu\right)=12\left(V^{*}\right)^{3}-9\left(V^{*}\right)^{2}(1+a+\nu)+2 V^{*}(1+a)(1+a+3 \nu)-\nu\left(2+a+2 a^{2}\right)
$$

and

$$
Q\left(V^{*} ; a, \nu\right)=-2\left(V^{*}\right)^{3}+\left(V^{*}\right)^{2}(1+a+3 \nu)-2 V^{*}(1+a) \nu+a \nu,
$$

we can summarise the conditions as follows.

The synchrony breaking bifurcation is subcritical if $N\left(V^{*} ; a, \nu\right)$ and $Q\left(V^{*} ; a, \nu\right)$ have 
opposite sign and supercritical if $N\left(V^{*} ; a, \nu\right)$ and $Q\left(V^{*} ; a, \nu\right)$ have the same sign.

Proof. As we are taking a limit to the parameter inside the sigmoidal function $k$, it is natural to look at the size of $k$ and its derivatives in this limit. The sigmoidal function $k$ is defined in eq. (4.18). In the work of Minai et al. [12] concise formulas for the derivatives of a sigmoidal function are derived, which are given by

$$
\begin{aligned}
k[x] & =\sigma(x ; p)=\frac{\alpha}{\alpha+\beta} \frac{1}{1+\exp (-p x)}, \\
k^{\prime}[x] & =p k(1-k), \\
k^{\prime \prime}[x] & =p^{2}\left(k(1-k)^{2}-k^{2}(1-k)\right), \\
k^{\prime \prime \prime}[x] & =p^{3}\left(k(1-k)^{3}-4 k^{2}(1-k)^{2}+k^{3}(1-k)\right) .
\end{aligned}
$$

In the limit for $p \rightarrow 0$, the terms without derivative of $k$ will dominate in the expression of eq. (4.13). We thus set $k=\frac{1}{2} \frac{\alpha}{\alpha+\beta}$ because $k \rightarrow \frac{1}{2} \frac{\alpha}{\alpha+\beta}$ if $p \rightarrow 0$. The resulting expression is

$$
\lim _{p \rightarrow 0}\left(H-\frac{F \hat{E}}{\hat{C}}\right)=\frac{1}{4}(-6)-\frac{1}{4} \frac{\left(-6 V^{*}+2(a+1)\right)^{2}}{\left(-3\left(V^{*}\right)^{2}+2(a+1) V^{*}-a-\frac{g}{2} \frac{\alpha}{\alpha+\beta}\right)} .
$$

The steady states $V^{*}$ solve eq. (4.19), which we can rewrite as follows

$$
\frac{V^{*}\left(V^{*}-a\right)\left(1-V^{*}\right)}{V^{*}-\nu}=g \cdot \frac{1}{2} \frac{\alpha}{\alpha+\beta}
$$

if we assume that $V^{*}-\nu \neq 0$. This expression can be substituted in eq. (4.26), resulting in

$$
\begin{aligned}
& \lim _{p \rightarrow 0}\left(H-\frac{F \hat{E}}{\hat{C}}\right)=\frac{-3}{2}-\frac{\left(-3 V^{*}+(a+1)\right)^{2}}{\left(-3\left(V^{*}\right)^{2}+2(a+1) V^{*}-a-\frac{V^{*}\left(V^{*}-a\right)\left(1-V^{*}\right)}{V^{*}-\nu}\right)} \\
= & \frac{-3}{2}-\frac{\left(-3 V^{*}+(a+1)\right)^{2}\left(V^{*}-\nu\right)}{\left(-3\left(V^{*}\right)^{2}\left(V^{*}-\nu\right)+2(a+1) V^{*}\left(V^{*}-\nu\right)-a\left(V^{*}-\nu\right)-V^{*}\left(V^{*}-a\right)\left(1-V^{*}\right)\right)} .
\end{aligned}
$$

For clarity define $P$ and $Q$ as the numerator and denominator of the second fraction, $\mathrm{SO}$

$$
\begin{aligned}
& P=\left(-3 V^{*}+(a+1)\right)^{2}\left(V^{*}-\nu\right), \\
& Q=-3\left(V^{*}\right)^{2}\left(V^{*}-\nu\right)+2(a+1) V^{*}\left(V^{*}-\nu\right)-a\left(V^{*}-\nu\right)-V^{*}\left(V^{*}-a\right)\left(1-V^{*}\right) .
\end{aligned}
$$

As we are interested in the sign of this coefficient, let us find expressions in terms of $V^{*}, a, \nu$ such that the coefficient is positive in this limit. We write the inequality in terms of $P$ and $Q$

$$
\lim _{p \rightarrow 0}\left(H-\frac{F \hat{E}}{\hat{C}}\right)>0 \Leftrightarrow \frac{-3}{2}-\frac{P}{Q}>0
$$


which can be rewritten as

$$
-\frac{2 P+3 Q}{2 Q}>0 \Leftrightarrow \frac{2 P+3 Q}{Q}<0 .
$$

We see that the coefficient is positive if the numerator $N:=2 P+3 Q$ and the denominator $Q$ have a different sign. The expressions can be written in terms of third-degree polynomials. We find that

$$
N\left(V^{*} ; a, \nu\right)=12\left(V^{*}\right)^{3}-9\left(V^{*}\right)^{2}(1+a+\nu)+2 V^{*}(1+a)(1+a+3 \nu)-\nu\left(2+a+2 a^{2}\right)
$$

and

$$
Q\left(V^{*} ; a, \nu\right)=-2\left(V^{*}\right)^{3}+\left(V^{*}\right)^{2}(1+a+3 \nu)-2 V^{*}(1+a) \nu+a \nu .
$$

We conclude that the coefficient $H-(F \hat{E}) / \hat{C}$ in limit $p \rightarrow 0$ is positive if $N\left(V^{*} ; a, \nu\right)<$ 0 and $Q\left(V^{*} ; a, \nu\right)>0$ or if $N\left(V^{*} ; a, \nu\right)>0$ and $Q\left(V^{*} ; a, \nu\right)<0$. In other words if $N\left(V^{*} ; a, \nu\right)$ and $Q\left(V^{*} ; a, \nu\right)$ have a different sign.

The coefficient $H-(F \hat{E}) / \hat{C}$ in limit $p \rightarrow 0$ is negative if $N\left(V^{*} ; a, \nu\right)>0$ and $Q\left(V^{*} ; a, \nu\right)>0$ or if $N\left(V^{*} ; a, \nu\right)<0$ and $Q\left(V^{*} ; a, \nu\right)<0$. In other words if $N\left(V^{*} ; a, \nu\right)$ and $Q\left(V^{*} ; a, \nu\right)$ have the same sign.

We remind ourselves that a positive value of the coefficient $H-(F \hat{E}) / \hat{C}$ corresponds to a subcritical pitchfork bifurcation from a synchronous steady state $V=V^{*}$ and a negative value corresponds to a supercritical pitchfork bifurcation.

Limit of fast changing input. Next, we consider the limit $p \rightarrow \infty$ in the expression for the sigmoidal function. Physically this corresponds to a fast change in input current when the membrane potential of the inputting neuron reaches the threshold potential. In more mathematical terms, the sigmoid approximates a Heaviside function. An example of a sigmoidal function for a large value of $p$ is shown in figure 4.4. For this limit of $p$ the higher order derivatives of $k$ are important.

In this limit $p \rightarrow \infty$ we find the result summarized in the following theorem. It turns out that the type of pitchfork bifurcation in this limit is only dependent on the sign of the coupling between the neurons.

ThEOREM 4.5. Consider the mutual dyad network of two identical neurons, described by simplified FitzHugh-Nagumo equations where $g \neq 0$ and $p \rightarrow \infty$. Assume that the synchronous steady state undergoes a synchrony breaking bifurcation. If the coupling between the neurons is excitatory, the type of pitchfork bifurcation is subcritical (a hard transition), and when the coupling is inhibitory, the type is supercritical (a soft transition).

Proof. Based on the behaviour of the sigmoid for $p \rightarrow \infty$, we consider two cases to find the steady states. In case that $V^{*}<V_{T}$, only the cubic part of eq. (4.14) remains and yields $V^{*}=0, a, 1$. For $V^{*}>V_{T}$ we find the expression

$$
V^{*}\left(V^{*}-a\right)\left(1-V^{*}\right)-g \cdot \frac{\alpha}{\alpha+\beta} \cdot\left(V^{*}-\nu\right)=0
$$


Taking the limit $p \rightarrow \infty$ in eq. (4.13) results in

$$
\lim _{p \rightarrow \infty}\left(H-\frac{F \hat{E}}{\hat{C}}\right)=\frac{1}{4} g \cdot\left(V^{*}-\nu\right) \cdot\left(k^{\prime \prime \prime}\left[V^{*}-V_{T}\right]-\frac{\left(k^{\prime \prime}\left[V^{*}-V_{T}\right]\right)^{2}}{k^{\prime}\left[V^{*}-V_{T}\right]}\right) .
$$

From the physical interpretation follows that conductivity $g>0$. The sign of $\left(V^{*}-\nu\right)$ depends on excitatory or inhibitory coupling to the input. We rewrite the expression involving the derivatives of $k$ using the results in eq. (4.23-4.25) from Minai et al. [12]. By using the form of these derivatives we can see that the expressions for $k^{\prime \prime \prime}$ and $\frac{\left(k^{\prime \prime}\right)^{2}}{k^{\prime}}$ are both of third order in $p$. We find that

$$
k^{\prime \prime \prime}-\frac{\left(k^{\prime \prime}\right)^{2}}{k^{\prime}}=-2 p^{3} k^{2}(1-k)^{2} .
$$

The resulting expression in the limit $p \rightarrow \infty$ states

$$
\lim _{p \rightarrow \infty}\left(H-\frac{F \hat{E}}{\hat{C}}\right)=-\frac{1}{2} g\left(V^{*}-\nu\right) p^{3} k^{2}(1-k)^{2} .
$$

To determine the sign of this expression we note that $g>0, p^{3}>0$ and $k^{2}(1-k)^{2}>0$, so the sign of $\lim _{p \rightarrow \infty}(H-(F \hat{E}) / \hat{C})$ is directly dependent on the sign of $V^{*}-\nu$. As $V^{*}$ is a (synchronous) steady state it fills the role of resting potential, which is present in the definition of $\nu$. If the coupling is excitatory, then $\nu>V^{*}$ or $V^{*}-\nu<0$, which results in $H-(F \hat{E}) / \hat{C}>0$. If the coupling is inhibitory, then $\nu<V^{*}$ or $V^{*}-\nu>0$ resulting in $H-(F \hat{E}) / \hat{C}<0$. From these results we can deduce that if the coupling is excitatory the type of pitchfork bifurcation is called subcritical and for inhibitory coupling the pitchfork bifurcation is called supercritical.

Acknowledgements. I would like to express my gratitude to Prof. Bob Rink for his patient guidance and useful critiques. His willingness to give his time has been very much appreciated. 


\section{References.}

[1] J. Carr. Application of Centre Manifold Theory, volume 35 of Applied Mathematical Sciences. Springer-Verlag New York, 1 edition, 1982.

[2] J. Carr. Center manifold. Scholarpedia, 1(12):1826, 2006. revision \#126955.

[3] T. Elmhirst and M. Golubitsky. Nilpotent hopf bifurcations in coupled cell systems. SIAM J. Appl. Dyn. Syst., 5:205-251, 2006.

[4] G.B. Ermentrout and D.H. Terman. Mathematical Foundations of Neuroscience, volume 35 of Interdisciplinary Applied Mathematics. Springer, 2010.

[5] R. FitzHugh. Mathematical models of threshold phenomena in the nerve membrane. Bull Math Biophys, 17(4):257-278, 1955.

[6] R. FitzHugh. Impulses and physiological states in theoretical models of nerve membrane. Biophys J, 1:445-466, 1961.

[7] M. Golubitsky and M. Krupa. Stability computations for nilpotent hopf bifurcations in coupled cell systems. Internat. J. Bifur. Chaos Appl. Sci. Engrg., 17:2595-2603, 2007.

[8] M. Golubitsky and C. Postlethwaite. Feed-forward networks, center manifolds, and forcing. Discrete Contin. Dyn. Syst., 32:2913-2935, 2012.

[9] M. Golubitsky and I. Stewart. Nonlinear dynamics of networks: the groupoid formalism. Bull. Amer. Math. Soc. (N.S.), 43(3):305-364, 2006.

[10] A.L. Hodgkin and A.F. Huxley. A quantitative description of membrane current and its application to conduction and excitation in nerve. J Physiol, 117(4):500 544, 1952.

[11] E. M. Izhikevich and R. FitzHugh. FitzHugh-Nagumo model. Scholarpedia, 1(9):1349, 2006. revision \#123664.

[12] A. I. Minai and R. D. Williams. On the Derivatives of the Sigmoid. Neural Networks, 6:845-853, 1993.

[13] J. Nagumo, S. Arimoto, and S. Yoshizawa. An active pulse transmission line simulating nerve axon. Proc IRE, 50(10):2061-2070, 1962.

[14] Nobelprize.org. The nobel prize in physiology or medicine 1963. Web, 2014. Accessed: 1 Jul 2016.

[15] B. Rink and J. Sanders. Coupled cell networks: semigroups, lie algebras and normal forms. Trans. Amer. Math. Soc., 367:3509-3548, 2015.

[16] B.W. Rink and J.A. Sanders. Amplified hopf bifurcations in feed-forward networks. SIAM J. Applied Dynamical Systems, 12(2):1135-1157, 2013.

[17] S.H. Strogatz. Nonlinear Dynamics and Chaos. Addison-Wesley, 1984.

[18] A. Vanderbauwhede. Dynamics Reported: A Series in Dynamical Systems and Their Applications, chapter Centre Manifolds, Normal Forms and Elementary Bifurcations, pages 89-169. Vieweg+Teubner Verlag, 1989. 